EPJ Web of Conferences 82, 01063 (2015)

DOI: $10.1051 /$ epjconf/20158201063

(C) Owned by the authors, published by EDP Sciences, 2015

\title{
Two-phase model of thermal decomposition of shale stratum
}

\author{
A.G. Knyazeva ${ }^{1,2}$, S.M. Martemiyanov ${ }^{2}$ and A.L. Maslov ${ }^{1,2, a}$ \\ ${ }^{1}$ Institute of Strength Physics and Materials Science of the Siberian Branch of the Russian Academy of \\ Sciences (ISPMS SB RAS), 2/4, Akademicheskii Av., Tomsk 634021, Russia \\ ${ }^{2}$ National Research Tomsk Polytechnic University (TPU), Lenin Av., 30, Tomsk 634050, Russia
}

There are various methods of oil shale thermal processing with the purpose to generate primary energy product - so-called shale gas - however, methods that do not require the extraction of rock to the surface are of special interest [1]. In these methods, shale gas is extracted directly from the stratum as it is heating up, in one way or another.

The aim of this work is to build a model of thermal decomposition of shale strata when they are heated by electromagnetic field.

The model takes into account the phenomenon of thermal conductivity, interphase heat and mass transfer, thermal effect of chemical reactions and gas flow in the pores.

Due to significant anisotropy of properties, shale stratum can be represented in the form of a solid porous plane, which has evenly distributed heat sources formed by electrodes, the distance between which $L$ is much larger than their radii $R_{0}$.

The geometry of the computational domain is shown in Fig. 1. Obviously, the challenge is to find the temperature distribution only in the destination area including one source with coordinates $(L / 2, L / 2)$.

Under the influence of an alternating electric field oil shale is heated by heat associated with its polarization, as well as by Joule heat caused by the conduction current.

When heated, solid phase reagent $A_{S}$ that forms the shale skeleton, decomposes into a solid product $B_{S}$ and gas $B_{G}$ coming into the pores. At the initial time some quantity of the gas $G_{R}$, which is identical in composition to the $B_{G}$, is already contained in the pores. In turn, when heated, the gas $G_{R}$ transforms into the gas $G_{p}$. Thus, the chemical reactions of the shale decomposition can be represented by the following conventional pattern:

$$
\begin{gathered}
A_{S} \stackrel{K_{1}}{\rightarrow} B_{S}+B_{g} \\
G_{R} \stackrel{K_{2}}{\rightarrow} G_{p}
\end{gathered}
$$

where $K_{1}, K_{2}$ - rates of reactions.

During decomposition the dominant mechanism of heating is subject to change: at first the effect is associated mainly with the dielectric losses, then due to accumulation of the product $B_{S}$ Joule heating is prevalent.

\footnotetext{
${ }^{a}$ Corresponding author: masloaleksey@rambler.ru
}

This is an Open Access article distributed under the terms of the Creative Commons Attribution License 4.0, which permits unrestricted use, distribution, and reproduction in any medium, provided the original work is properly cited. 


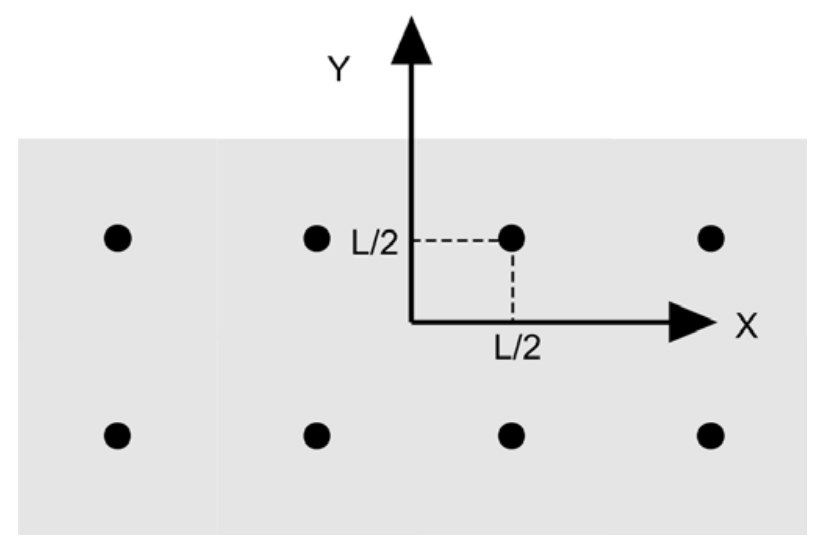

Figure 1. Illustration for the problem formulation.

Calorimetric measurements of shale thermal decomposition [2] have shown that the summarized thermal effect of shale decomposition reaction is positive, and at the initial stage of kerogen decomposition endothermic effects are taking place. On further temperature raising the exothermic stage starts, coinciding with the secondary gasification. We assume that thermal effect of solid phase decomposition is negative, and that gaseous product reacts with heat release, and besides, exothermal heat effect is prevalent.

The equation of thermal conductivity for solid phase is as follows:

$$
c_{s} \rho_{s} \frac{\partial T_{s}}{\partial t}=\lambda_{s} \nabla \cdot\left(\nabla_{k} T_{s}\right)+Q_{1} W_{1}+\Phi_{s}-\frac{\alpha_{T}}{1-\varsigma}\left(T_{s}-T_{g}\right),
$$

where $T_{S}, c_{S}, \rho_{S}$ - temperature, heat capacity and density of the solid phase, accordingly; $t$ - time, $x, y-$ spatial coordinates; $\lambda_{s}$ - coefficient of heat conductivity of the solid phase, $W_{1}$ - chemical source (drain) of heat, $\Phi_{s}$ - heat source associated with the effect of electromagnetic field on solid phase (depends on the location of the electrodes, their radii, characteristics of the electric field) [3], $\alpha_{T}$ - coefficient of heat transfer between gas and solid phase; $\varsigma$ - porosity; $T_{g}$ - gas temperature.

Thermal effect of alternating electromagnetic field consists of a resistance and dielectric losses:

$$
\Phi_{S}=\eta_{B_{S}} \sigma_{1} E^{2}+\left(1-\eta_{B_{S}}\right) \varepsilon_{1} \varepsilon_{0} \Gamma E^{2} \operatorname{tg} \delta_{1}
$$

where $E^{2}=E_{x}^{2}+E_{y}^{2}$ - squared electric field intensity; $E=E(x, y), \sigma_{1}$ - conductivity, $f$ - frequency, $\varepsilon_{1}$-permittivity of hard rock, $\varepsilon_{0}$ - dielectric constant, $\operatorname{tg} \delta_{1}$ - angle tangent (ratio) of dielectric losses.

Shares of the products of the solid phase reaction are defined by the following equations:

$$
\begin{gathered}
\frac{d \eta_{B_{S}}}{d t}=k_{1}\left(1-\eta_{B_{G}}-\eta_{B_{S}}\right) \exp \left(-\frac{E_{1}}{R T_{S}}\right) v_{S}, \\
\frac{d \eta_{B_{G}}}{d t}=k_{1}\left(1-\eta_{B_{G}}-\eta_{B_{S}}\right) \exp \left(-\frac{E_{1}}{R T_{S}}\right) v_{g}-\frac{\alpha_{m}}{1-\varsigma}\left(\eta_{B_{G}}-\eta_{G}\right)
\end{gathered}
$$

where $k_{1}$ - pre-exponential factor, $E_{1}$ - activation energy of the solid phase reaction, $R$ - gas constant, $v_{S}, v_{g}$ - stoichiometric coefficients for the solid phase reaction.

Equation of heat transfer in the gas phase is as follows:

$$
c_{g} \rho_{g} \frac{d T_{g}}{d t}=Q_{2} W_{2}+\Phi_{g}+\frac{\alpha_{T}}{\varsigma}\left(T_{s}-T_{g}\right),
$$




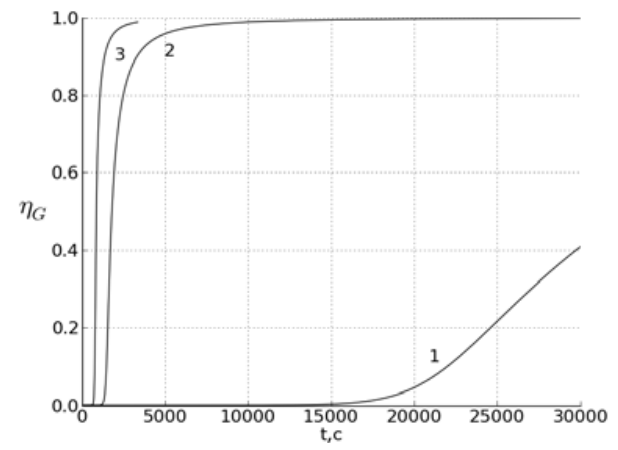

Figure 2. Dynamics of gaseous product share at varying potential (values are taken in the vicinity of the electrode). $1-\varphi=20000$ B., $2-\varphi=30000$ B., $3-\varphi=35000$ B. $\varsigma=0.25, f=10 \mathrm{kHz}$.

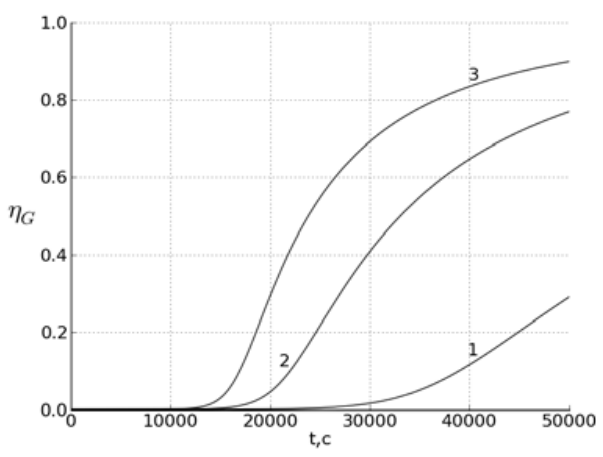

Figure 3. Dynamics of gaseous product share at varying porosity (values are taken in the vicinity of the electrode). $1-\varsigma=0.1,2-\varsigma=0.25,3-\varsigma=0.4 . \varphi=20000 \mathrm{~B} ., f=10 \mathrm{kHz}$.

where $c_{g}, \rho_{g}$ - gas heat capacity and density; $\Phi_{g}=\eta_{G} \sigma_{2} E^{2}+\left(1-\eta_{G}\right) \varepsilon_{2} \varepsilon_{0} \Gamma E^{2} \operatorname{tg} \delta_{2}-$ heat source associated with the action of electromagnetic field on gas; $\sigma_{2}$ - conductivity, $\varepsilon_{2}-$ gas permittivity; $\operatorname{tg} \delta_{2}$ - angle tangent (ratio) of dielectric losses; $\frac{d \ldots}{d t}=\frac{\partial \ldots}{\partial t}+v_{x} \frac{\partial \ldots}{\partial x}+v_{y} \frac{\partial \ldots}{\partial y}, v_{x}, v_{y}-$ components of gas velocity vector $\boldsymbol{v}_{g}$.

Total gas phase reaction is exothermic and conforms to the following schema: $G_{R} \rightarrow G_{p}$. The proportion of gaseous reactant is changed by the following law:

$$
\frac{d \eta_{G}}{d t}=-k_{2} \eta_{G} \exp \left(-\frac{E_{2}}{R T_{g}}\right) v_{G}+\frac{\alpha_{m}}{\varsigma}\left(\eta_{B G}-\eta_{G}\right)
$$

where $k_{2}$ - pre-exponential factor; $E_{2}$ - activation energy of the gaseous phase reaction; $v_{G}-$ stoichiometric coefficient, $\frac{d \ldots}{d t}=\frac{\partial \ldots}{\partial t}+v_{x} \frac{\partial \ldots}{\partial x}+v_{y} \frac{\partial \ldots}{\partial y}$.

Equation for condition of a gas, which could not be regarded as perfect, in the first approximation is as follows:

$$
d p_{g}=K_{T}\left(\alpha_{T} d T_{g}+\alpha_{G} d \eta_{g}\right),
$$

where $p_{g}$ - gas pressure; $K_{T}$ - volumetric isothermal elastic module, $\alpha_{T}$ - coefficient of thermal expansion, $\alpha_{T}$ - concentration expansion factor.

Gas velocity was calculated by the Darcy's law:

$$
\boldsymbol{v}_{g}=-K_{f} \nabla p_{g}
$$




\section{EPJ Web of Conferences}

where $K_{f}=\frac{K}{\eta}, K$ - coefficient of filtration, $\eta$-dynamic viscosity, whence it follows:

$$
\boldsymbol{v}_{g}=-K_{f} K_{T}\left(\alpha_{T} \nabla T+\alpha_{G} \nabla \eta_{G}\right) .
$$

The problem is solved numerically by using an implicit scheme of splitting by coordinates and coordinate-wise run.

Example of calculation for gaseous product accumulation is shown in Figs. 2, 3:

\section{References}

[1] James G. Speight. Shale Oil Production Processes. Elsevier (2012)

[2] William I. Stuart and John H. Levy. Thermal properties of carbonaceous oil shales from the Nagoorin and Condor deposits. Fuel, 66, pp. 493-498 (1987)

[3] Knyazeva A. G., Lopatin V. V., Martemiyanov S. M., Maslov A. L., Han Wei. Modeling of shale underground heating by electromagnetic field // Proceedings of higher educational establishments. Physics - Vol. 54, - No. 11/3. - pages 5-11 (2011) 